\title{
HEART
}

\section{Ischaemic mitral regurgitation: mechanisms and diagnosis}

Thomas H Marwick, Patrizio Lancellotti and Luc Pierard

Heart 2009 95: 1711-1718

doi: 10.1136/hrt.2007.135335

Updated information and services can be found at:

http://heart.bmj.com/content/95/20/1711.full.html

Topic collections Articles on similar topics can be found in the following collections

\author{
Valvular heart disease (13 articles) \\ Education in Heart (341 articles) \\ Drugs: cardiovascular system (22480 articles) \\ Echocardiography (1621 articles) \\ Acute coronary syndromes (1331 articles) \\ Mitral valve disease (115 articles) \\ Clinical diagnostic tests (18660 articles) \\ Epidemiology (4706 articles)
}

Notes

To order reprints of this article go to:

http://heart.bmj.com/cgi/reprintform

To subscribe to Heart go to:

http://heart.bmj.com/subscriptions 


\title{
Ischaemic mitral regurgitation: mechanisms and diagnosis
}

\author{
Thomas H Marwick, ${ }^{1}$ Patrizio Lancellotti, ${ }^{2}$ Luc Pierard ${ }^{2}$
}

- Supplemental video footage is available online only at http:// heart.bmj.com/content/vol95/ issue20

${ }^{1}$ University of Queensland, Princess Alexandra Hospital, Brisbane, Australia; ${ }^{2}$ University Hospital Sart Tilman, Liege, Belgium

Correspondence to:

Professor T Marwick, University of Queensland School of Medicine, Princess Alexandra Hospital, Ipswich Road, Brisbane, Old 4102, Australia; t.marwick@uq.edu.au
Ischaemic mitral regurgitation (MR) is defined as $M R$ caused by changes of left ventricular structure and function related ultimately to ischaemia. However, the acute manifestation of MR following infarction (which usually presents as a haemodynamic crisis) is related to rupture or stretching of the papillary muscle, and is normally categorised with complications of infarction. The term ischaemic $M R$ is usually understood to relate to chronic $M R$, occurring $>2$ weeks after infarction and in the absence of structural mitral valve disease. In terms of pathogenesis, this should be considered a disease of abnormal left ventricular (LV) shape and function with a valvular manifestation.

The frequency in ischaemic MR varies according to the technique used for its detection (being more common in echocardiographic than angiographic studies), the management of the patients (more common in non-revascularised patients), the timing post-myocardial infarction (MI) (more common early, before medical treatment is optimised), and infarct size. These selection influences were avoided in the study of a geographically defined MI incidence cohort in which MR was identified at 30 days in $50 \%$ of 773 patients, $12 \%$ of which were moderate or severe, and in whom the detection by physical examination was unreliable. ${ }^{1}$

Ischaemic $\mathrm{MR}$ is not only a common but also a serious finding. The community based study of ischaemic MR among 30 day survivors of MI showed moderate or severe MR to be associated with a threefold increase in the risk of heart failure and a 1.6-fold increased risk of death at 5 year follow-up independent of age, gender, ejection fraction (EF), and Killip class. ${ }^{1}$ Mortality was increased even with mild MR. ${ }^{2}$

\section{MECHANISMS OF ISCHAEMIC MR}

The mechanism of ischaemic MR requires a combination of both leaflet tethering and reduction of closing forces (table 1). The difficulty posed by separating the role of each component has been addressed by in vitro work suggesting that apical and posterior displacement of the papillary muscles are the dominant features. These effects are all amplified in the setting of a dilated annulus. ${ }^{3}$

\section{Valvular and subvalvular changes}

Disturbances of posterior leaflet displacement (asymmetric pattern)

The classic pattern of ischaemic MR involves a posterior wall motion abnormality with regional remodelling, leading to posterolateral and apical displacement of the (posterior) papillary muscle, the apical component of which appears to be the most important. This regional remodelling appears to be related to the regional scar burden. ${ }^{2}$ As the papillary muscle contributes chordae to both leaflets, the consequences are: (1) displacement of the posterior leaflet posteriorly; (2) development of a hockey stick deformity of the anterior leaflet due to tethered secondary chordae; and (3) displacement of the mitral coaptation point posteriorly (causing an asymmetric shape). The consequence is anterior leaflet override with a posterior $M R$ jet (fig 1). Usually this arises from the centre of the valve, but the process may disproportionately involve the medial commissure (fig 2), perhaps reflecting papillary muscle elongation.

Left ventricular dilatation (symmetric pattern) Global enlargement of the left ventricle (LV) usually occurs in association with increased sphericity and annular dilatation, and all contribute to MR. The coaptation point of the MV is moved apically, with a large tenting area, ${ }^{4}$ and both leaflets are involved to a similar degree, causing a central jet (fig 3). This circumstance is typically associated with large infarct size-typically an anterior infarction or multiple infarctions. ${ }^{5}$ Asymmetric and symmetric mechanisms may become mixed, as remodelling of the LV with MR due to papillary muscle displacement may add global to regional remodelling.

\section{Annular dilatation}

Annular dilatation and flattening is a frequent accompaniment to global LV remodelling. Loss of the typical saddle shape of the annulus reduces leaflet curvature and thereby increases leaflet stress. ${ }^{6}$ However, patients with lone atrial fibrillation, who develop mitral annular dilation in the absence of ventricular abnormalities, show less MR than those with dilated cardiomyopathy, even after correction for annulus size. ${ }^{7}$ The normal mitral leaflet area is more than double the area of the annulus, indicating a significant reserve before annular enlargement leads to non-coaptation. Annular enlargement is generally uniform, and experimental work has indicated annular enlargement $>1.75$-fold would be required to engender MR. ${ }^{8}$ Thus, the independent contribution of annular enlargement to ischaemic MR has perhaps been overstated. Its role appears to be adjunctive, 
Table 1 Typical features of asymmetric and symmetric patterns of ischaemic mitral regurgitation

\begin{tabular}{lll}
\hline & Asymmetric & Symmetric \\
\hline Leaflet tethering & PML tethered towards posterior wall & Both tethered towards apex \\
$>$ MR jet direction & Eccentric, posterior & Usually central \\
$>$ Tenting area & Increased & Greatest \\
$>$ Annulus & Minor change & Dilated and flattened \\
$>$ LV remodelling & Regional & Global \\
$>$ MI site & Inferior & Anterior/multiple \\
$>$ CAD extent & $\mathrm{RCA} / \mathrm{Cx}$ & Multivessel \\
\hline
\end{tabular}

CAD, coronary artery disease; $C x$, circumflex coronary artery; LV, left ventricular; Ml, myocardial infarction; MR, mitral regurgitation; $\mathrm{PML}$, posterior papillary muscle; $\mathrm{RCA}$, right coronary artery.

with evidence that this increases MR at different levels of papillary muscle displacement. ${ }^{3}$ The exception to this statement relates to basal inferoposterior infarcts, which may cause asymmetric annular dilatation. ${ }^{9}$

\section{Papillary muscle discoordination}

The papillary muscles are the main contributors to acute ischaemic MR. Although traditionally incriminated in chronic ischaemic MR, the importance of associated regional LV dysfunction has been recognised for nearly 40 years. ${ }^{10}$ In rare instances, papillary muscle scarring may lead to elongation and mitral prolapse.

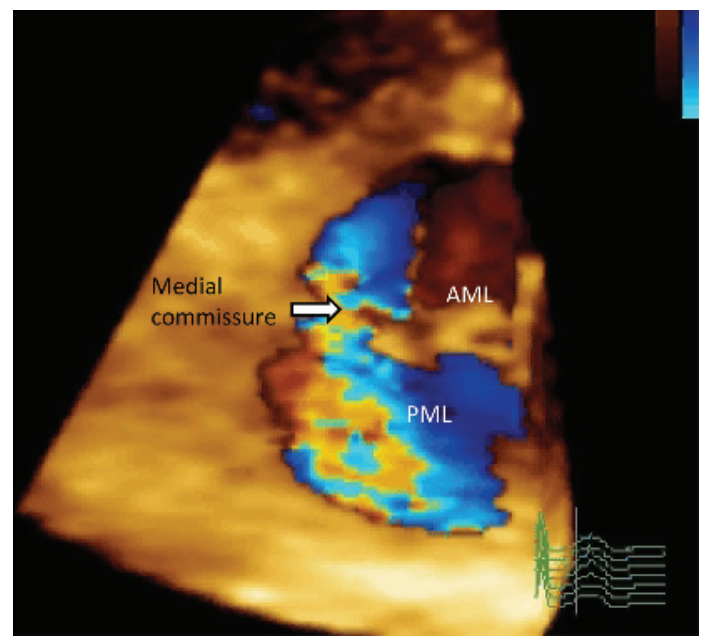

Figure 2 Ischaemic mitral regurgitation with disproportionate involvement of the medial commissure. $\mathrm{AML}$, anterior mitral leaflet; PML, posterior mitral leaflet.

The contribution of papillary muscle dyssynchrony has been more recently recognised as an important component of LV dyssynchrony. In left bundle branch block (LBBB), mechanical activation occurs first in the segment adjacent to the posterior papillary muscle and is delayed in the segment with the anterolateral papillary muscle insertion.
Figure 1 Ischaemic mitral regurgitation (MR) following inferoposterior myocardial infarction. End systolic freeze frames in apical views from a three dimensional

echocardiogram (lower right) show how posteriorly directed ischaemic mitral regurgitation arises from remodelling of the posterolateral wall, with displacement of the posterior papillary muscle (arrows). The resulting apical tethering of the posterior leaflet (dashed circle) causes posteriorly directed MR (lower left).

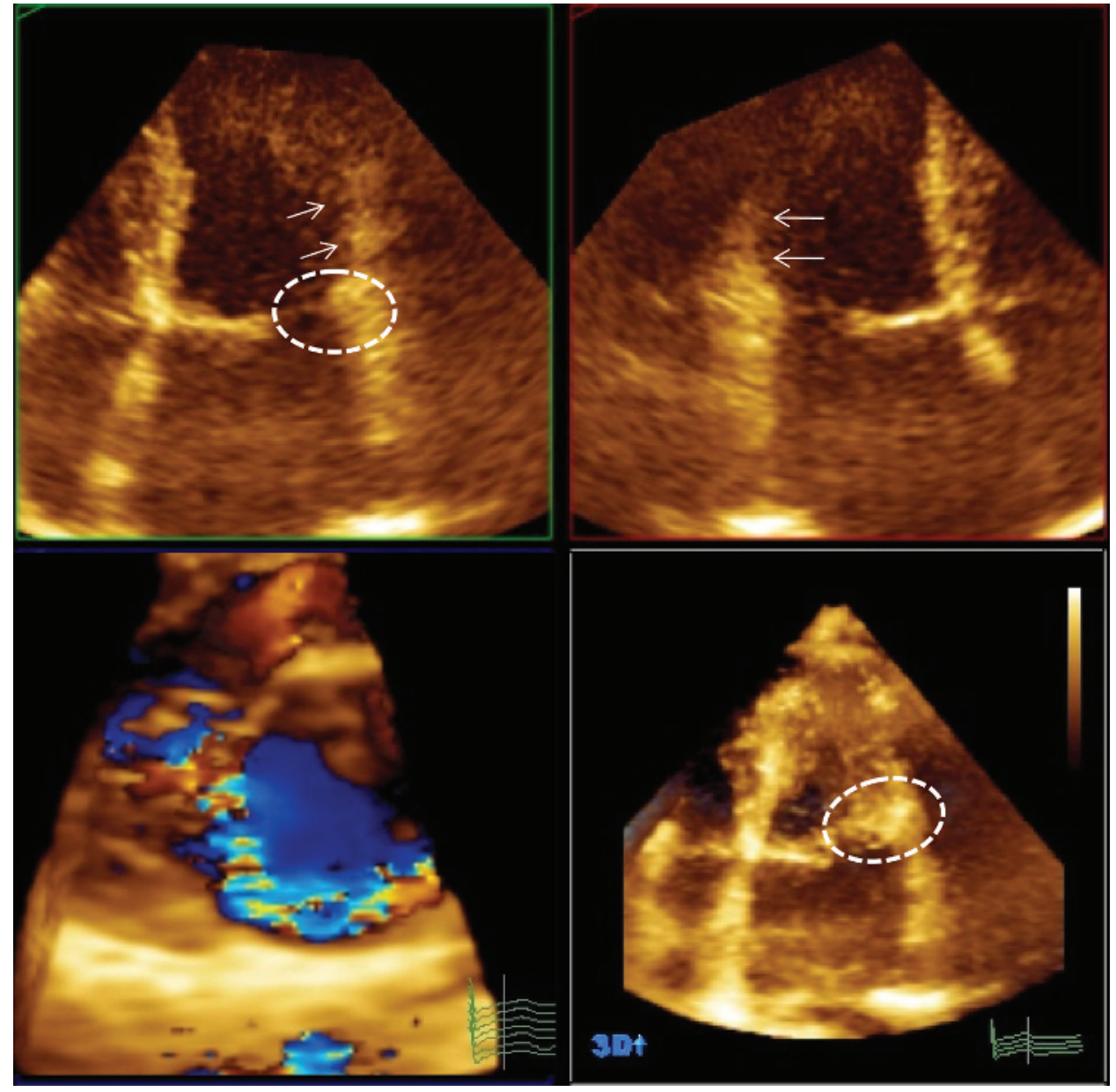




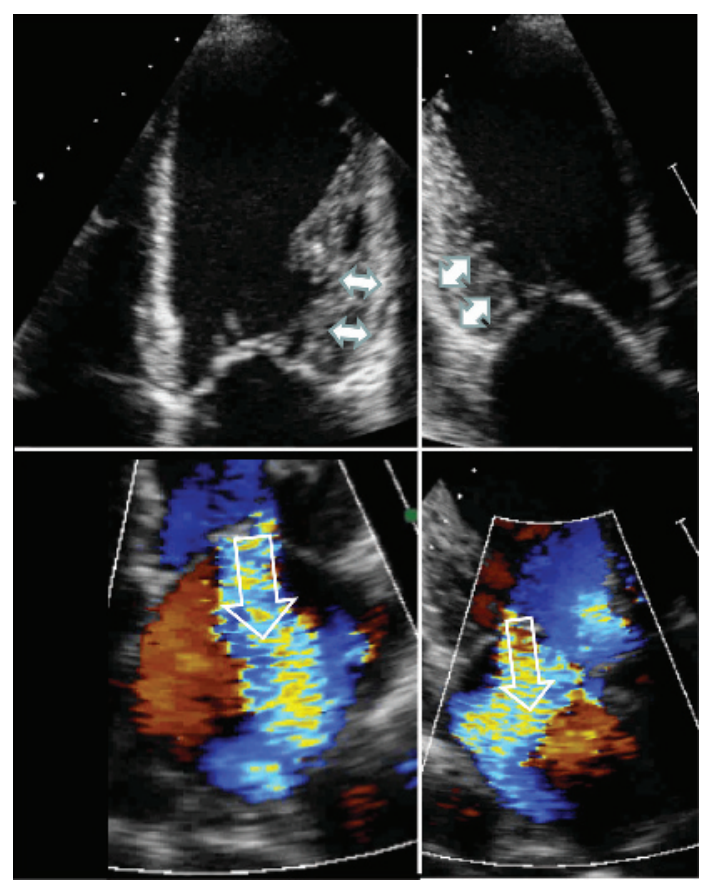

Figure 3 Ischaemic mitral regurgitation (MR) following left anterior descending (LAD) and right coronary artery (RCA) territory infarctions-end-systolic freeze frames show the septum is thinned but there is preserved thickening in the posterior wall (double headed arrows). Left ventricular remodelling has caused displacement of both papillary muscles with failure of coaptation of the mitral leaflets and central MR (open arrow).

With cardiac resynchronisation therapy (CRT), the interpapillary muscle activation time delay is shortened and its magnitude of change, which varies substantially from patient to patient, is significantly correlated with the decrease in mitral regurgitant fraction. ${ }^{11}$ Reductions in this interpapillary activation delay (fig 4) correlate well with reduction of $\mathrm{MR}$ following CRT.

\section{Closing forces}

Impairment of LV contractile function is associated with all of the morphological changes listed above. However, it is unusual for LV dysfunction to cause $\mathrm{MR}$ in the absence of LV remodelling. ${ }^{3}$ The severity of ischaemic $M R$ is a dynamic balance between tethering and closing forces (table 2). ${ }^{12}$ These interactions result in potentially significant variation of ischaemic MR over time (fig 5). The presence of only mild global dysfunction with preserved closing forces may attenuate the severity of MR that might otherwise arise from the regional changes that cause non-coaptation. Treatments (myocardial revascularisation, CRT, medical therapy, and exercise) which influence closing pressure may significantly modulate ischaemic MR.

\section{Myocardial revascularisation}

Revascularisation does not necessarily reverse ischaemic $M R$. In a study of coronary bypass surgery in 136 patients with moderate ischaemic $M R$, half showed an improvement in MR, but resolution of MR occurred in only $9 \%$, and $40 \%$ had residual moderate or severe MR. ${ }^{13}$ The effects of revascularisation on closing pressure are likely to depend on the presence and extent of viable myocardium. There is a link between the amount of scarring (defined by contrast enhanced magnetic resonance imaging (MRI)) and the severity of $M R,{ }^{14}$ and one study showed that improved posterior wall function after thrombolysis was associated with a lower frequency of $M R .{ }^{15}$ The role of viable myocardium in ischaemic $M R$ is probably important but unproven.

\section{CRT}

The effects of CRT may not relate purely to papillary muscle resynchronisation, and also relates to improvement in $\mathrm{dP} / \mathrm{dt}$ with CRT therapy. ${ }^{16}$ This contribution to improved closing forces is evidenced by the acute haemodynamic effect of CRT, before the occurrence of reverse remodelling. The acute decrease in mitral effective regurgitant orifice is directly correlated to the increase in LV systolic performance $(\mathrm{dP} / \mathrm{dt})$, which counteracts the increased tethering forces that impair mitral valve competence. ${ }^{16}{ }^{17}$ In addition, diastolic $\mathrm{MR}$ is relieved by shortening atrioventricular (AV) delay. ${ }^{18}$ After successful CRT implantation, MR improves in most patients, remains unchanged in about $25 \%$, and worsens in a relatively small number of patients.

\section{Medical treatment}

Treatment is an important contributor to the variation of ischaemic MR over time (fig 5). Dobutamine, which is widely used in the acute setting to support LV dysfunction, rarely provokes $\mathrm{MR}$ and more commonly causes no change or reduces the severity of $M R$, both in groups with impaired and preserved LV function. ${ }^{19}$ Other medical treatments have not been well studied. Most patients can be expected to show a reduction of $\mathrm{MR}$ in response to vasodilation, but these results were heterogenous and no more associated with dynamic than fixed regurgitant valves. ${ }^{20}$

\section{Exercise}

Exercise is an important cause for variability of ischaemic $M R$, irrespective of the baseline severity of $M R .^{21}$ The normal response to exercise involves vasodilation (reducing tethering forces), global contractile reserve (increasing closing forces), and regional contractile reserve (normalising function or shape in the posterior wall). However, ischaemia, a hypertensive response to exercise or loss of contractile reserve may all lead to worsening MR with exercise. ${ }^{21}$ An increase of effective regurgitant orifice $(E R O) \geqslant 13 \mathrm{~mm}^{2}$ carries adverse prognostic implications, ${ }^{22}$ possibly through recurrence of this process causing remodelling, or exercise induced heart failure. The determinants of exercise induced changes of MR include systolic annular area, tenting area, wall motion score, and the degree of apical displacement of the mitral valve. 
Figure 4 Inter-papillary activation delay, shown with colour tissue Doppler.

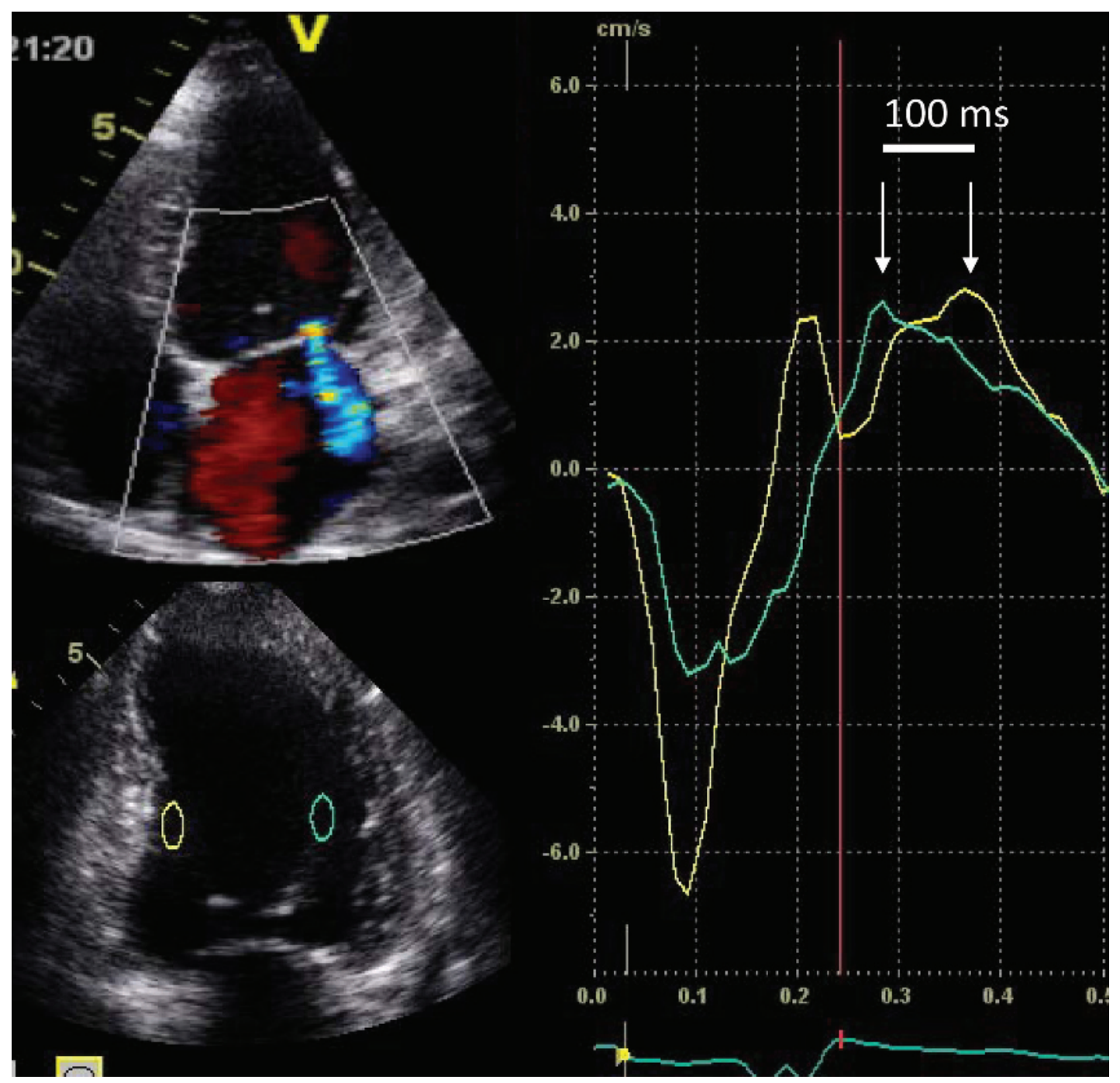

\section{DIAGNOSIS OF ISCHAEMIC MR}

The clinical signs of $M R$ and its severity are unreliable in ischaemic MR. Irrespective of imaging technique, the report needs to address findings related to LV morphology and ejection fraction, mitral valve morphology, and the severity of $M R$, as these features govern the indications for surgery in the current guidelines. ${ }^{23}$

\section{LV morphology and function}

As ischaemic MR is fundamentally a ventricular disease, the global report should include observations about LV volume and shape (including sphericity). The regional evaluation should include comments about wall thinning, wall motion abnormalities (and if possible, their contractile

Table 2 Tethering and closing forces in ischaemic mitral regurgitation

\begin{tabular}{ll}
\hline Tethering forces & Closing forces \\
\hline Papillary muscle displacement & Reduced LV contractility \\
LV dilatation and sphericity & LV dyssynchrony \\
Annular dilatation & Papillary muscle dyssynchrony \\
& $>$ Reduced annular contraction \\
\hline
\end{tabular}

LV, left ventricle. reserve), and perhaps a marker of scar thickness (using strain or contrast).

The role (if any) of measuring LV synchrony is controversial. Current guidelines for CRT are based on symptom status, LV function and ORS duration, and the reliability of current measures of mechanical synchrony do not seem to justify their incorporation into decision making. ${ }^{24}$ On the other hand, observational studies demonstrate an improvement in moderate ischaemic $M R$ and mitral tethering with CRT, ${ }^{25}$ proportionate to the degree of inter-papillary delay, measured in the short axis view.

\section{Mitral valve morphology}

The mitral findings should include consideration of the morphology of the valve as well as MR jet direction. A posterior directed jet is usually associated with eccentric valve involvement, while a central jet marks symmetrical involvement (table 1).

Despite the normal structure of mitral leaflets in ischaemic MR, the displacement of the leaflets into the LV leads to a number of characteristic changes of valve morphology. Annular dimension should be $<35 \mathrm{~mm}$ (in the anteroposterior axis). A number 
Figure 5 Temporal variation of ischaemic mitral regurgitation (MR) severity. This patient presented following a posterior wall myocardial infarction, and at the time of presentation had severe MR with a prominent tenting angle. Following transfer to our facility, in the course of which the patient was treated with inotrope and left ventricular volumes decreased, the MR almost resolved with reduction of tenting angle. After a percutaneous intervention she still had moderate mitral regurgitation, emphasising the role of inotropes in acutely reducing ischaemic MR.

Figure 6 Measurements of abnormal mitral morphology in ischaemic mitral regurgitation. Tenting area $2.6 \mathrm{~cm}^{2}$, coaptation distance $1 \mathrm{~cm}$, angle $35^{\circ}$.
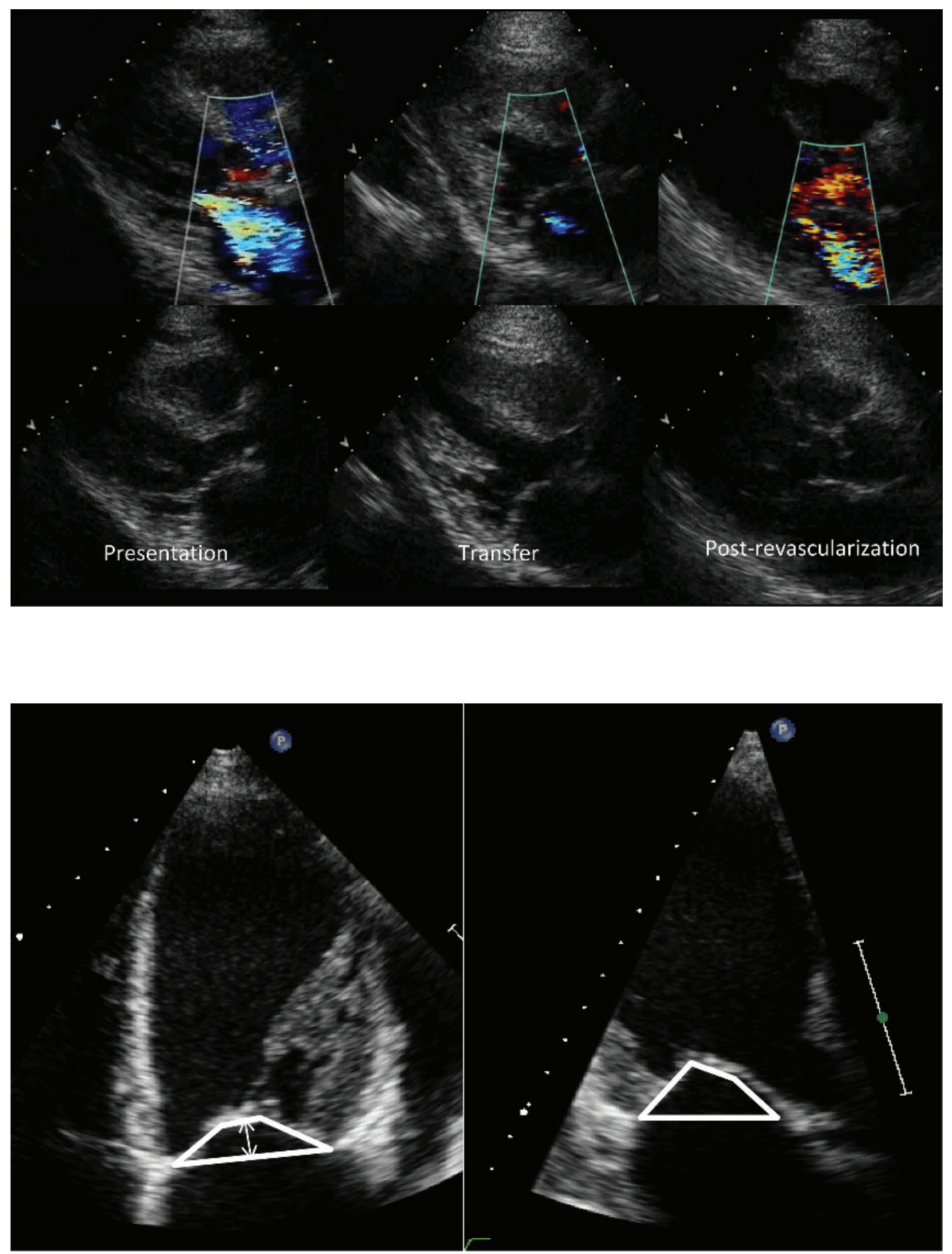

Important contributors to ischaemic MR

- Structural

- Disturbances of posterior leaflet displacement (asymmetric pattern)

- Left ventricular dilatation (symmetric pattern)

- Annular dilatation

- Functional

- Papillary muscle discoordination

- Closing pressure: viability, ischaemia, medical treatment, exercise of anatomic measurements can be made that reflect the pathophysiology of ischaemic MR, including tenting area, leaflet angles, coaptation depth, bending distance and leaflet length (fig 6). Greater degrees of morphologic disturbance are predictive of greater likelihood of persistence of MR following mitral annuloplasty, with the optimal cut-offs for distinguishing patients with persistent $\mathrm{MR}$ being a coaptation distance of $>0.6 \mathrm{~cm}$, a tenting area of $>2.5 \mathrm{~cm}^{2}$, or a posterior leaflet angle $>45^{\circ} .{ }^{26}$ Measures of tenting volume 


\section{Education in Heart}

Figure 7 Application of three dimensional echocardiography to the measurement of tenting volume.
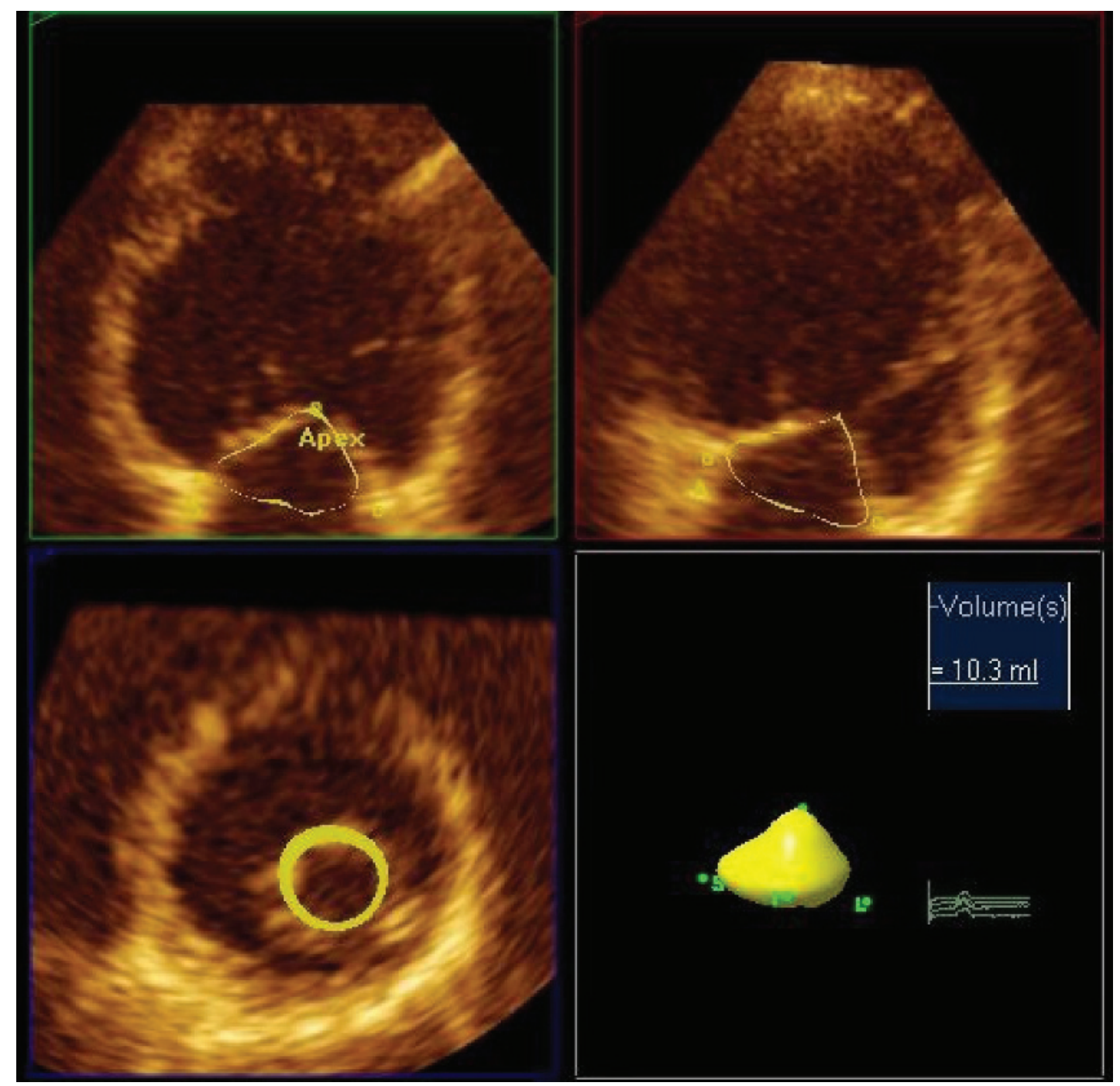

are now readily available using three dimensional echocardiography (fig 7)

These features of mitral valve configuration may vary from time to time according to loading conditions, and it is not clear as to whether they provide incremental information to standard Doppler measures for the assessment of $M R$ severity.

\section{The imaging report in ischaemic MR}

- Left ventricular (LV) morphology and function

- LV volume and shape (including sphericity)

- Wall thinning, wall motion abnormalities

- Contractile reserve

- Scar thickness

- LV synchrony (controversial)

- Mitral valve morphology

- MR jet direction (eccentric vs symmetrical LV dysfunction)

- Annular dimension

- Tenting area, leaflet angles, coaptation depth

- Severity

- Beware inhomogeneity of MR and variability and complexity of jet morphology

\section{Severity}

MR severity may be difficult to assess in ischaemic regurgitation. In part, this relates to variations in $M R$ severity during systole. Typically, the severity of $M R$ is greatest at the beginning and end of systole and least in mid-systole, when the LV pressure is maximal, LV volume is reduced, and the mitral leaflets are pushed back into the annular plane (fig 8). ${ }^{27}$ This phenomenon has important implications with respect to the use of regurgitant orifice area as an index of $M R$, implying that this should be averaged through systole. The use of volumetric methods for calculating regurgitant volume may also avoid errors due to inhomogeneity of MR. Measurement of vena contracta width may be a preferable means of assessing $M R$ severity, although this is equally influenced by changes in LV pressure and dimensions. There is evidence that three dimensional echocardiography is superior to two dimensional techniques for the measurement of vena contracta, especially with eccentric jets. ${ }^{28}$

The second contributor to the difficulty of assessing MR severity in ischaemic MR relates to the variability and complexity of jet morphology. Irregularly shaped and multiple jets, caused by a 
Figure 8 Non-uniform progression of ischaemic mitral regurgitation through systole (arrows demonstrate narrowing of the vena contracta). Accurate quantitation requires averaging at multiple time points.

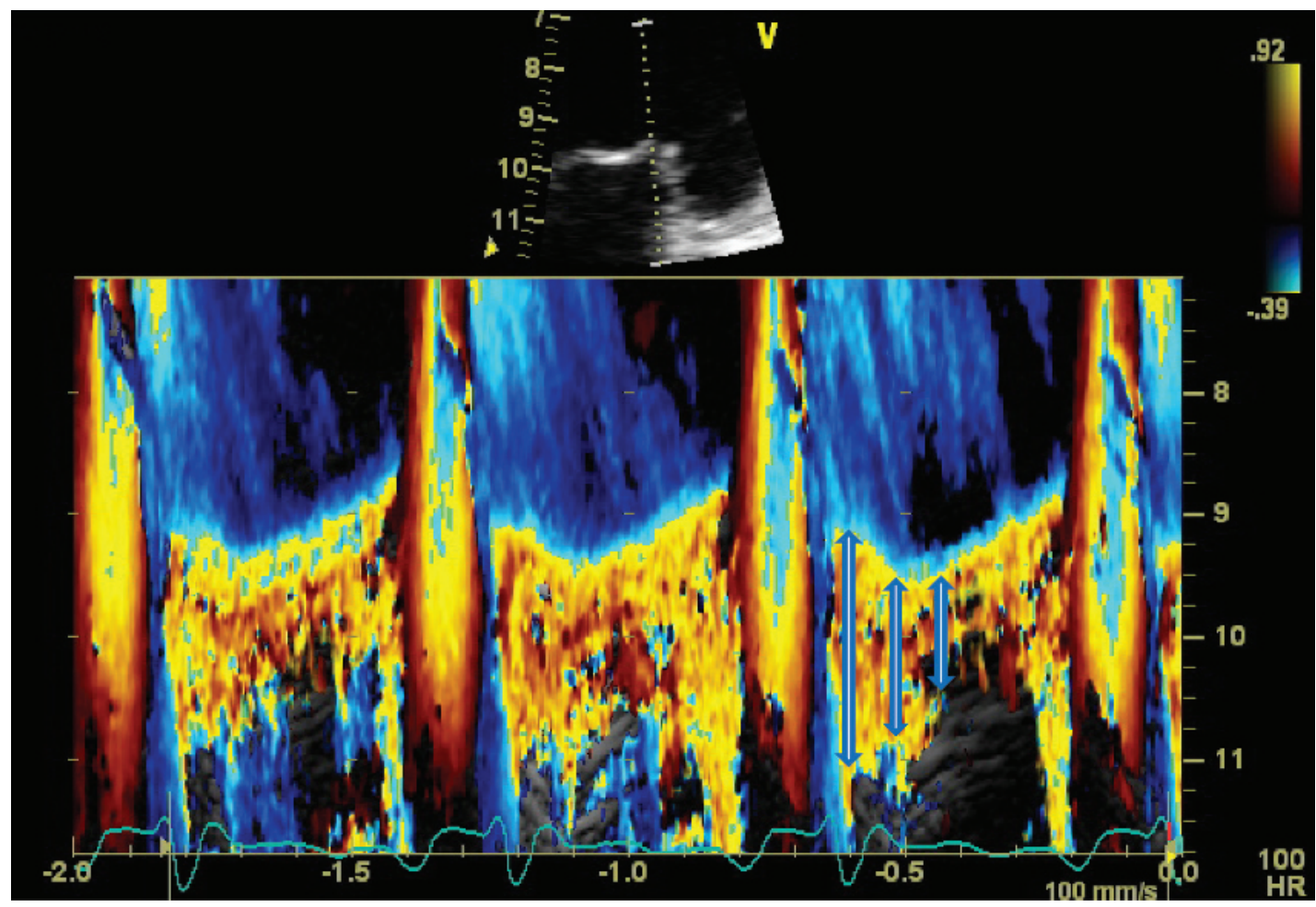

variety of mechanisms, ${ }^{29}$ pose problems for the proximal isovelocity surface area approach. ${ }^{4}$ It is important to remember that relatively moderate ischaemic MR has an adverse prognosis, and therefore particular caution should be paid to recognition of moderate lesions.

\section{CONCLUSIONS}

Ischaemic $M R$ is relatively common and carries an adverse prognosis. Although therapeutic strategies are currently limited, recognition of the predominant mechanisms may in the future allow rational therapies, targeted towards specific mechanisms.

\section{You can get CPD/CME credits for Education in Heart}

Education in Heart articles are accredited by both the UK Royal College of Physicians (London) and the European Board for Accreditation in Cardiologyyou need to answer the accompanying multiple choice questions (MCQs). To access the questions, click on BMJ Learning: Take this module on BMJ Learning from the content box at the top right and bottom left of the online article. For more information please go to: http://heart.bmj.com/misc/education.dtl

- RCP credits: Log your activity in your CPD diary online (http://www. rcplondon.ac.uk/members/CPDdiary/index.asp)—pass mark is $80 \%$.

- EBAC credits: Print out and retain the BMJ Learning certificate once you have completed the MCOs - pass mark is $60 \%$. EBAC/ EACCME Credits can now be converted to AMA PRA Category 1 CME Credits and are recognised by all National Accreditation Authorities in Europe (http://www.ebac-cme.org/ newsite/?hit=men02).

Please note: The MCQs are hosted on BMJ Learning - the best available learning website for medical professionals from the BMJ Group. If prompted, subscribers must sign into Heart with their journal's username and password. All users must also complete a one-time registration on BMJ Learning and subsequently log in (with a BMJ Learning username and password) on every visit.
Funding: Supported in part by a Program grant from the National Health and Medical Research Council, Canberra, Australia

Competing interests: In compliance with EBAC/EACCME guidelines, all authors participating in Education in Heart have disclosed potential conflicts of interest that might cause a bias in the article. The authors have no competing interests.

Provenance and peer review: Commissioned; not externally peer reviewed.

\section{REFERENCES}

1. Bursi F, Enriquez-Sarano M, Nkomo VT, et al. Heart failure and death after myocardial infarction in the community: the emerging role of mitral regurgitation. Circulation 2005;111:295-301

2. Grigioni $\mathbf{F}$, Enriquez-Sarano $\mathrm{M}$, Zehr $\mathrm{KJ}$, et al. Ischemic mitral regurgitation: long-term outcome and prognostic implications with quantitative Doppler assessment. Circulation 2001:103:1759-64.

- A touchstone in this field, this large follow-up study defines the poor outcome associated with even moderate ischaemic MR.

3. He S, Fontaine AA, Schwammenthal E, et al. Integrated mechanism for functional mitral regurgitation: leaflet restriction versus coapting force: in vitro studies. Circulation 1997;96:1826-34.

4. Watanabe N, Ogasawara $Y$, Yamaura $Y$, et al. Quantitation of mitral valve tenting in ischemic mitral regurgitation by transthoracic real-time three-dimensional echocardiography. J Am Coll Cardiol 2005:45:763-9.

5. Kaul S, Spotnitz WD, Glasheen WP, et al. Mechanism of ischemic mitral regurgitation. An experimental evaluation. Circulation 1991;84:2167-80.

6. Salgo IS, Gorman JH III, Gorman RC, et al. Effect of annular shape on leaflet curvature in reducing mitral leaflet stress. Circulation 2002;106:711-7.

7. Otsuji Y, Kumanohoso T, Yoshifuku S, et al. Isolated annular dilation does not usually cause important functional mitral regurgitation: comparison between patients with lone atrial fibrillation and those with idiopathic or ischemic cardiomyopathy. J Am Coll Cardiol 2002;39:1651-6.

8. He S, Lemmon JD Jr, Weston MW, et al. Mitral valve compensation for annular dilatation: in vitro study into the mechanisms of functional mitral regurgitation with an adjustable annulus model. J Heart Valve Dis 1999;8:294-302. 
9. Gorman JH III, Gorman RC, Plappert T, et al. Infarct size and location determine development of mitral regurgitation in the sheep model. J Thorac Cardiovasc Surg 1998;115:615-22.

10. Mittal AK, Langston M Jr, Cohn KE, et al. Combined papillary muscle and left ventricular wall dysfunction as a cause of mitral regurgitation. An experimental study. Circulation 1971;44:174-80.

11. Kanzaki H, Bazaz R, Schwartzman D, et al. A mechanism for immediate reduction in mitral regurgitation after cardiac resynchronization therapy: insights from mechanical activation strain mapping 1. J Am Coll Cardiol 2004:44:1619-25.

- This important study documents the role of papillary muscle synchrony as a contributor to functional MR and demonstrates the role of improving mechanical synchrony in reducing MR.

12. Levine RA, Schwammenthal E. Ischemic mitral regurgitation on the threshold of a solution: from paradoxes to unifying concepts. Circulation 2005;112:745-58.

13. Aklog L, Filsoufi F, Flores KQ, et al. Does coronary artery bypass grafting alone correct moderate ischemic mitral regurgitation? Circulation 2001;104:168-75

14. Srichai MB, Grimm RA, Stillman AE, et al. Ischemic mitral regurgitation: impact of the left ventricle and mitral valve in patients with left ventricular systolic dysfunction 1. Ann Thorac Surg 2005;80:170-8.

- There are limited data connecting myocardial status with the response of ischaemic MR. This paper examines the contribution of scar burden in this setting.

15. Tenenbaum A, Leor J, Motro M, et al. Improved posterobasal segment function after thrombolysis is associated with decreased incidence of significant mitral regurgitation in a first inferior myocardial infarction. J Am Coll Cardiol 1995;25:1558-63.

16. Breithardt $\mathbf{0 A}$, Sinha AM, Schwammenthal E, et al. Acute effects of cardiac resynchronization therapy on functional mitral regurgitation in advanced systolic heart failure 8. J Am Coll Cardiol 2003;41:765-70.

17. Vinereanu D, Turner MS, Bleasdale RA, et al. Mechanisms of reduction of mitral regurgitation by cardiac resynchronization therapy. J Am Soc Echocardiogr 2007;20:54-62.

18. Nof E, Glikson M, Bar-Lev D, et al. Mechanism of diastolic mitral regurgitation in candidates for cardiac resynchronization therapy. Am J Cardiol 2006; 97:1611-4.

19. Heinle SK, Tice FD, Kisslo J. Effect of dobutamine stress echocardiography on mitral regurgitation. J Am Coll Cardiol 1994;25:122-7.

20. Kizilbash AM, Willett DL, Brickner ME, et al. Effects of afterload reduction on vena contracta width in mitral regurgitation. J Am Coll Cardiol 1998;32:427-31.
21. Lancellotti $\mathbf{P}$, Lebrun F, Pierard LA. Determinants of exerciseinduced changes in mitral regurgitation in patients with coronary artery disease and left ventricular dysfunction. J Am Coll Cardiol 2003:42:1921-8.

22. Lancellotti P, Troisfontaines P, Toussaint AC, et al. Prognostic importance of exercise-induced changes in mitral regurgitation in patients with chronic ischemic left ventricular dysfunction Circulation 2003;108:1713-7.

23. Vahanian A, Baumgartner $\mathrm{H}, \mathrm{Bax} \mathrm{J}$, et al. Guidelines on the management of valvular heart disease: the Task Force on the Management of Valvular Heart Disease of the European Society of Cardiology. Eur Heart J 2007;28:230-68.

- Ischaemic MR is the result of a dynamic process. References 22 and 23 systematically examine the role of exercise as well as the prognostic implications of ischaemic MR.

24. Gorcsan J III, Abraham T, Agler DA, et al. Echocardiography for cardiac resynchronization therapy: recommendations for performance and reporting - a report from the American Society of Echocardiography Dyssynchrony Writing Group endorsed by the Heart Rhythm Society. J Am Soc Echocardiogr 2008;21:191-213.

25. Ypenburg C, Lancellotti P, Tops LF, et al. Acute effects of initiation and withdrawal of cardiac resynchronization therapy on papillary muscle dyssynchrony and mitral regurgitation. J Am Coll Cardiol 2007;50:2071-7.

26. Magne J, Pibarot $\mathrm{P}$, Dagenais $\mathrm{F}$, et al. Preoperative posterior leaflet angle accurately predicts outcome after restrictive mitral valve annuloplasty for ischemic mitral regurgitation. Circulation 2007:115:782-91.

27. Schwammenthal E, Chen C, Benning F, et al. Dynamics of mitral regurgitant flow and orifice area. Physiologic application of the proximal flow convergence method: clinical data and experimental testing. Circulation 1994;90:307-22.

- An important work that links surgical outcome of restrictive annuloplasty with the details of mitral valve geometry.

28. Little SH, Pirat B, Kumar R, et al. Three-dimensional color Doppler echocardiography for direct measurement of vena contracta area in mitral regurgitation: in vitro validation and clinical experience. J Am Coll Cardiol Img 2008;1:695-704.

29. Song JM, Kim MJ, Kim YJ, et al. Three-dimensional characteristics of functional mitral regurgitation in patients with severe left ventricular dysfunction: a real-time three-dimensional colour Doppler echocardiography study. Heart 2008;94:590-6.

- Ischaemic MR is often associated with eccentric MR jets. This paper explores the role of three dimensional echocardiography in understanding the shape of the MR jet. An appreciation of this complexity facilitates the evaluation of MR severity. 